\title{
Correlation between Functional Staging and DDLS in Primary Open Angle Glaucoma Patients in Vietnam
}

\author{
Van Anh Thi BUI ${ }^{1, *}$, Thom Thi VU' ${ }^{2}$ and Tung Thanh HOANG ${ }^{3}$ \\ ${ }^{1}$ Vietnam National Institute of Ophthalmology, Vietnam \\ ${ }^{2}$ School of Medicine and Pharmacy, Vietnam National University, Ha Noi, Viet Nam \\ ${ }^{3}$ Hanoi Medical University, Dong Da, Ha Noi, Vietnam
}

('Corresponding author's e-mail: buivananh@vnio.vn)

Received: 6 March 2019, Revised: 28 November 2019, Accepted: 29 December 2019

\begin{abstract}
Clinically, there are many systems of visual field imaging systems. The agreement between glaucoma function and appearance is still uncertain. This study was conducted to compare visual field staging classifications (as function staging) with Disc Damage Likelihood Scale (DDLS as appearance staging) in Primary Open Angle Glaucoma (POAG). A cross-section study was performed using 55 patients (90 eyes) examined and treated by the Vietnam National Institute of Ophthalmology from 9/2016 - 9/2017. The MD (Mean Deviation), PSD (Pattern Standard Deviation), and VFI (Visual Field Index) indexes were recorded and were used to classify glaucoma staging according to categories of eGSS (enhanced Glaucoma Severity Staging), mGSS (modified Glaucoma Severity Staging), HPA (HodappParrish-Aderson), AGIS (Advanced Glaucoma Intervention Study), and DDLS. Using SPSS 16.0 software, statistical significance was tested with proper tests of Chi-square test, Fisher exact test, Kappa (to assess the agreement-disagreement), and Spearman Rank (to assess the level of correlation). Mean VFI were $-11.49 \mathrm{~dB}$ (MD), $5.85 \mathrm{~dB}$ (PSD), and 74.2\% (VFI). Compared to DDLS, all systems had slight agreement $(\mathrm{K}<0.2)$. The disagreement percentage was highest in eGSS $(70 \%)$, followed by mGSS $(50$ $\%$ ) and HPA (48.9 \%). In terms of definitive diagnosis, HPA and mGSS showed a substantial agreement $(\mathrm{K}>0.6)$ with AGIS, which was higher than that between eGSS and AGIS (0.773 and 0.75 vs 0.399), with $p<0.001$. In terms of staging detection, the agreement between HPA, mGSS, and AGIS was substantial $(\mathrm{K}>0.6)$, while that between eGSS and AGIS was fair $(\mathrm{K}<0.4)$. In conclusion, mGSS and HPA tended to show stronger agreement with standard classifications than eGSS. mGSS should be used in clinical practice and research.
\end{abstract}

Keywords: DDLS, eGSS, mGSS, HPA, Glaucoma

\section{Introduction}

It has been found that damage to about 25 - $30 \%$ of retinal ganglion cells begins to appear as damage in visual field tests [1]. However, a number of other studies have shown that, while there are many cases of injury in the visual field, the ganglion cell layer has no injury. Although a series of quantitative models of the correspondence between functional injury and structure have been proposed as Hood's model - Karrdon, Harwerth and colleagues, Drasdo and colleagues and Stick - Stick models [9]. However, this relationship is still a potential research topic. Until now, counting the number of incidents of, or accurately determining the level of, retinal ganglion cell damage to help assess the extent of physical injury is still an area that medicine has not fully explored. Additionally, the use of DDLS classification is sometimes difficult to implement, due to difficulty in image storage or subjective analysis [2]. Therefore, finding a functional classification almost similar to the structure classification may provide doctors with more tools to predict glaucoma stages. 
http://wjst.wu.ac.th

Since the American Medical Association's severity of visual field classification system was first published in 1958, there have been more than 20 classification systems published, but none of these is considered the gold standard in assessing the severity of visual field damage [10]. In 2016, a group of Japanese authors published modified Glaucoma Staging System (mGSS) based on the VFI threshold improved from Mills' Bascom Palmer GSS [5]. After that, the classification received attention from experts because of its ability to accurately evaluate and the use of simple categories. However, is mGSS really better than previous classifications like Hodapp-Parrish-Anderson (HPA) and enhanced Glaucoma Staging System (eGSS)? Also, is the mGSS correlated to DDLS classification better than HPA and eGSS? To clarify these 2 research hypotheses, we conducted research on 3 glaucoma classification systems: HPA, eGSS, and mGSS. In this paper, we focus on analyzing the consensus between visual field damage classification by eGSS, mGSS, and HPA with DDLS to find out the most appropriate visual field classification to help predict the extent of structural damage.

\section{Materials and methods}

\section{Patients}

We enrolled 90 eyes of 55 patients of the Glaucoma Department of the Vietnam National Institute of Ophthalmology (VNIO) from 2016 to 2017, all of which had been diagnosed with Primary Open Angle Glaucoma (POAG). Patients from 18 to 80 years old, who had ability to complete visual field tests and to cooperate with the checking of their eyes, were selected. The research proposal was approved by the Scientific and Ethics committee of the Vietnam National Institute of Ophthalmology, coding number 160/QD-BVMTW.

\section{Sampling methods}

Visual field printout: a visual field test checks for eyes affected with glaucoma was performed using Humphrey perimeter, 24-2 SITA tests (Swedish Interactive Threshold Algorithm Standard) for determining definitive diagnosis, glaucoma severity diagnosis, and VFI value. The visual field printouts had reliable index, and loss of fixation, false positive error, and false negative error of more than $20 \%$ were excluded from study.

The severity stage of eyes with glaucoma: based on the visual field printouts, these were classified by using HPA, eGSS and mGSS scales simultanously, as shown in Tables 1 and 2 and Figure 1. The HPA scale is presented in Table 1 below.

Table 1 HPA scale (Hodapp et al., 1999).

\begin{tabular}{llll}
\hline & MD (dB) & PSD & \multicolumn{1}{c}{ At 5 from Fixation Point } \\
\hline Early & $\geq-6$ & $<25 \%$ of points at $<5 \%$ and & No point at $<15 \mathrm{~dB}$ \\
& & $<15 \%$ of points at $<1 \%$ & \\
Moderate & $\geq-12$ & $25-50 \%$ of points at $<5 \%$ and & No point at $0 \mathrm{~dB}$; at least 1 point in 1 \\
& & $15-25 \%$ of points at $<1 \%$ & hemifield at $<15 \mathrm{~dB}$ \\
Severe & $<-12$ & $>50 \%$ of points at $<5 \%$ and & $\begin{array}{l}\text { At least } 1 \text { point at } 0 \mathrm{~dB} \text {; both hemifield } \\
\text { have point at }<15 \mathrm{~dB}\end{array}$ \\
\hline
\end{tabular}


eGSS uses the relationship between MD and PSD to determine 7 stages of glaucoma severity, including 0 - 5 stages, as shown in Figure 1:

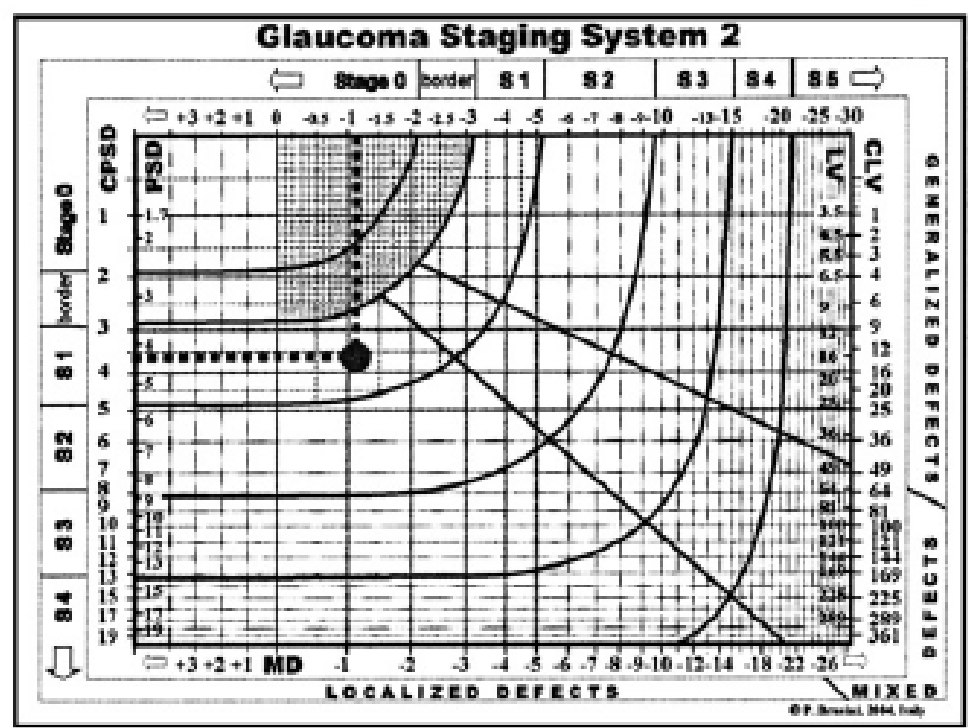

Figure 1 eGSS (evolutive Glaucoma Staging System, 2013).

mGSS uses VFI to divide Glaucoma stages into 6 stages based on the criteria of Anderson and Patella, as in Table 2.

Table 2 mGSS scale

\begin{tabular}{llllll}
\hline Stage Number & VFI (\%) & Stage Severity & Stage Number & VFI (\%) & Stage Severity \\
\hline 0 & - & No damage & 3 & $43-62 \%$ & Advanced \\
1 & $\geq 82 \%$ & Early & 4 & $23-42 \%$ & Severe \\
2 & $63-81 \%$ & Moderate & 5 & $<22 \%$ & Blind \\
\hline
\end{tabular}

To evaluate the consistency and the correlation of the 3 different classification systems, HPA (with 3 stages: early, moderate, and severe), eGSS (with 7 stages: 0 - 5), and mGSS (with 6 stages: 0 - 5), our team proposed an Integrated Classification (IC), bringing all systems into a common frame of reference, with 4 levels for convenience in surveying systems.

Table 3 Integrated classification of HPA, eGSS, and mGSS systems.

\begin{tabular}{clccc}
\hline \multicolumn{2}{c}{ Integrated Classification (IC) } & HPA & eGSS & mGSS \\
\hline IC1 & Normal & & 0 & 0 \\
(Normal) & & Borderline & 1 \\
IC2 & Early/Lesion Minimum & 1 & 2 \\
(Early) & & & 2 & 3 \\
IC3 & Moderate & Moderate & 3 & 4 \\
(Moderate) & & & 4 & 5 \\
IC4 & Severe/Absolute & Severe & 5 & \\
(Severe) & & & & \\
\hline
\end{tabular}


Eye examination: all eyes with glaucoma were slit-lamp checked at the Glaucoma Department-VNIO. Corneal performance, the anterior chamber, and the lens were thoroughly checked to exclude any secondary glaucoma. Damage to the optic nerve head was reported and classified into a stage using DDLS (Table 4).

Table 4 Classification of the trends of disc lesions (Spaeth, 2005).

\begin{tabular}{cclll}
\hline & \multicolumn{1}{c}{ Thinnest Width of Optic Nerve Fiber Layer } \\
\hline & Stage & \multicolumn{1}{c}{$\begin{array}{c}\text { Small Disc } \\
<\mathbf{1 . 5} \mathbf{~ m m}\end{array}$} & \multicolumn{1}{c}{$\begin{array}{c}\text { Average Disc } \\
\mathbf{1 . 5} \mathbf{- 2} \mathbf{~ m m}\end{array}$} & $\begin{array}{c}\text { Large Disc } \\
\mathbf{2} \mathbf{~ m m}\end{array}$ \\
\hline Glaucoma & 1 & $\geq 0.5$ & $\geq 0.4$ & $\geq 0.3$ \\
Risk & 2 & $0.4-0.49$ & $0.3-0.39$ & $0.2-0.29$ \\
& 3 & $0.3-0.39$ & $0.2-0.29$ & $0.1-0.19$ \\
& 4 & $0.2-0.29$ & $0.1-0.19$ & $<0.1$ \\
\hline Damage as & 5 & $0.1-0.19$ & $<0.1$ & 0 with $<45^{\circ}$ \\
Glaucoma & 6 & $<0.1$ & 0 with $<45^{\circ}$ & 0 with $46-90^{\circ}$ \\
& 7 & 0 with $<45^{\circ}$ & 0 with $46-90^{\circ}$ & 0 with $91-180^{\circ}$ \\
\hline Glaucoma & 8 & 0 with $46-90^{\circ}$ & 0 with $91-180^{\circ}$ & 0 with $181^{\circ}-270^{\circ}$ \\
Progression & 9 & 0 with $91-180^{\circ}$ & 0 with $181^{\circ}-270^{\circ}$ & 0 with $>270^{\circ}$ \\
and Severity & 10 & 0 with $>180^{\circ}$ & 0 with $>270^{\circ}$ & \\
\hline
\end{tabular}

Eyes with glaucoma with visual field results at the IC1 stage were considered to be of normal visual field (VF), and IC2-4 stages were considered to be of abnomal VF. Eyes with glaucoma wth disc damage at stages 1 - 4 in DDLS were considered to be normal discs, and the rest abnomal discs.

\section{Statistical analysis}

Data were analyzed by SPSS 16.0 software. Analysis of variance (ANOVA) of one factor and 2 factors; Chi-square tests $(\chi 2)$ were applied to the corresponding cases. $p$-value of less than 0.05 was viewed as a statistically significant difference.

Data processing: Vulnerable eye rate, according to each classification DDLS, HPA, eGSS, and mGSS, was statistically, proportionally, and consensus analyzed, according to the Kappa algorithm (agreement level). K and $p$ of Chi-square, Fisher exact (assessment of relevance), and Spearman Rank (assessment of correlation level) tests were performed. The level of statistical significance $p<0.05$ was used. Data were processed by SPSS 16.0 software. Descriptive statistics included calculation of average value, standard deviation (for quantitative data) and frequency, and percentage (with qualitative data). Inference statistics were done through Chi-square, Kappa, and Spearman Rank tests. Kappa test was used to evaluate the consistency between 2 different disease classification systems with the same number of phases through coefficient $\mathrm{K}: \mathrm{K}<0$ (no consistency); $\mathrm{K}=0.01-0.2$ (weak consistency); $\mathrm{K}=0.21-0.4$ (moderate consistency); $\mathrm{K}=0.411-0.6$ (average consistency); $\mathrm{K}=0.61-0.8$ (high consistency); $\mathrm{K}=$ $0.81-0.99$ (fully consistent). Spearman Rank evaluated the correlation of 2 sequences of rankings through coefficient $p$. This factor takes the value from -1 to +1 . The closer the value of $p$ to 1 is, the closer the correlation is. The rating levels of $p$ are as follows: the absolute value of $p$ is $0-0.19$ : the degree of correlation is very weak; from 0.2 to 0.39 : weak correlation; from 0.4 to 0.59 : average correlation level; from 0.6 to 0.79 : tight correlation; from 0.8 to 1 : the correlation is very tight. 


\section{Results and discussion}

\section{Patient's characteristics}

In our study, $38 \%$ patients were middle-aged (40 - 60 years old), $36 \%$ were young patients (less than 40 years old), and $26 \%$ were old patients (more than 60 years old). The male/female ratio was 21/34. These distributions could be found in some other studies [5,8]. The visual field results were recorded in 90 printouts of 55 glaucoma patients in the study with average MD $-11.49 \pm 8.67 \mathrm{~dB}((-29.55)$ - $(-1.4))$, average PSD $5.85 \pm 4.08 \mathrm{~dB}(1.02-14)$, and average VFI $74.2 \pm 28.56 \%$. The distribution of VFI in normal and abnormal eyes, according to the scales, is seen in Table 5.

Table 5 Distribution of VFI in normal and abnormal eyes

\begin{tabular}{llcccc}
\hline & & HPA & eGSS & mGSS & DDLS \\
\hline \multirow{5}{*}{ Normal } & MD & -4.06 & -2.58 & -4.05 & \\
& PSD & 1.8 & 1.51 & 1.78 & \\
& VFI & 97.75 & 99.36 & 97.84 & \\
& No & $32(35,6 \%)$ & $11(12,2 \%)$ & $31(34,4 \%)$ & $74(82,2 \%)$ \\
\hline \multirow{5}{*}{ Abnormal } & PSD & -15.59 & -12.73 & -15.41 & \\
& MD & 8.08 & 6.45 & 7.99 & \\
& VFI & 60.93 & 70.49 & 61.51 & \\
& No & $58(64,4 \%)$ & $79(87.8 \%)$ & $59(65,6 \%)$ & $16(17,8 \%)$ \\
\hline
\end{tabular}

The normal rate in eGSS classification was less than in HPA and mGSS classification, even though it was not statistically significant, simultaneously, guided MD value average was less, and VFI average was higher, in normal eyes, according to eGSS classification. This difference showed that the sensitivity of eGSS seems higher when compared to HAP or mGSS.

Comparing HPA, eGSS, and mGSS to DDLS in the diagnosis of glaucoma

Analyzing data using the Kappa algorithm, we found that all 3 systems had low homogeneity with DDLS in the diagnosis of glaucoma $(\mathrm{K}<0.2)$, even though uniformity was significant with $p<0.05$, as presented in Table 6. This showed that, in the diagnosis of disease, although all classification methods could lead to homogeneity in the case of the disease being clear (with uniformity being statistically significant), in the case of doubt, the cut-off value of identity of disease was very different between systems, making the level of uniformity low.

Table 6 Distribution of VFI in normal and abnormal eyes

\begin{tabular}{llcccccc}
\hline & & \multicolumn{2}{c}{ HPA } & \multicolumn{2}{c}{ eGSS } & \multicolumn{2}{c}{ mGSS } \\
\hline DDLS & Yes & Normal & Abnormal & Normal & Abnormal & Normal & Abnormal \\
& No & 31 & 15 & 0 & 16 & 1 & 15 \\
& \multicolumn{2}{c}{$\mathrm{K}=0.176(p=0.007)$} & $\mathrm{K}=0.058(p=0.1)$ & $\mathrm{K}=0.167(p=0.009)$ \\
& \multicolumn{2}{l}{ Test 2 $(p=0.001)$} & Test 2 $(p=0.059)$ & Test 2 $(p=0.002)$ \\
\hline
\end{tabular}

When using the $\mathrm{X}^{2}$ algorithm for analysis, we found that the HPA and mGSS visual field classifications had a significant relationship with the DDLS classification, while eGSS had no significant relationship with DDLS $(p=0.059)$. This was further confirmed when we found that the disagreement ratio between eGSS and DDLS was very high, with $70 \%$. This value was calculated by formula 
http://wjst.wu.ac.th

disagreement between eGSS and DDLS ratio $=(0$ eye (normal on eGSS, abnormal on DDLS $)+63$ eyes (abnormal on eGSS, normal on DDLS)) / 90 eyes. The disagreement ratios between DDLS and HPA and mGSS were lower, at 50 and $48.9 \%$, respectively. Thus, based on the classification of eGSS, it was completely impossible to predict the vulnerability of nerve endings. At the same time, the HPA and mGSS classification methods seemed to be more accurate in diagnosing disease.

Paired Comparison between HPA and DDLS; eGSS and DDLS; mGSS and DDLS

Figure 2 shows that, in the HPA classification, $13 \%$ of normal visual field cases had damage to the optic nerve head, and $15 \%$ had severe visual field injuries but with no existing neurological damage. In the HPA classification, glaucoma damage was divided into 3 simple stages: early, moderate, and severe. Classification was based primarily on 2 criteria: the width of the damage assessed by the MD, and the number of injury points on the local deviation scale; the location of injury involved being close to the point of fixation. Because of not assessing the depth of localized lesions, sometimes HPA classification could make the level of injury become lighter than the deep lesions of the nerve fiber bundle shown in the DDLS classification [12]. This might be the cause of disagreement between these 2 classification systems.

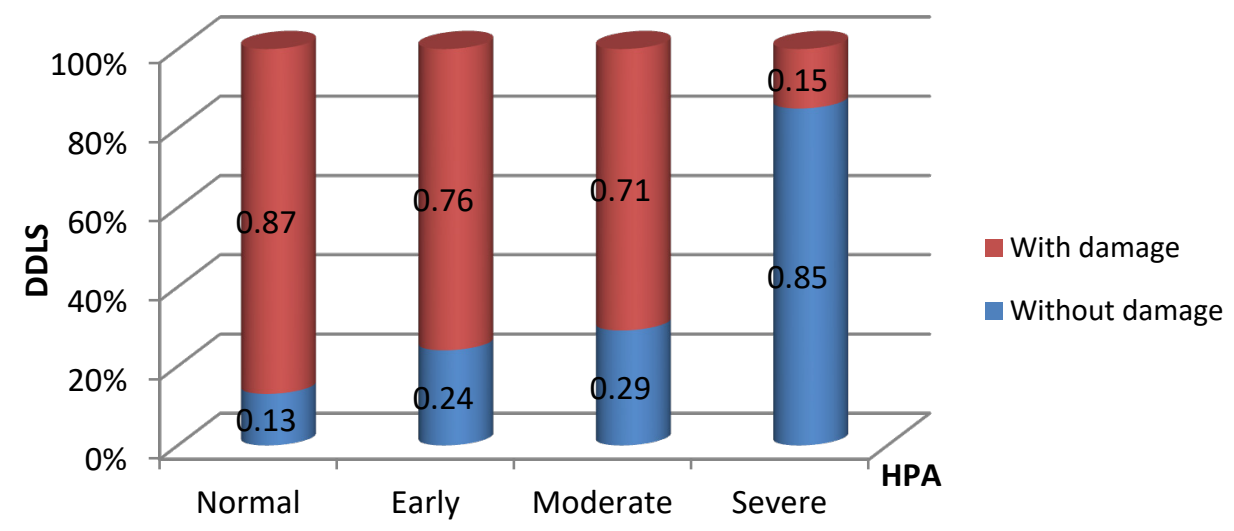

Figure 2 Comparison between HPA and DDLS.

eGSS or GSS 2 was announced by Brusini and Filacorda in 2006, based on 2 visual field indicators, MD and PSD, to classify the stage when using SITA strategy [4]. eGSS classification was optimized by charting disease progression between time points. The main advantage of eGSS was its simplicity, convenient for daily clinical practice. However, the disadvantages of eGSS could be affected by false injuries and short- and long-term fluctuations; it did not provide information about the location of injury. Therefore, different lesions at different times could be classified as being the same [13]. In this study, we found that up to $35 \%$ of cases diagnosed with severe glaucoma stage, according to eGSS, were normal manifestations of the optic nerve head. This rate was highest compared to mGSS (11\%) and HPA $(15 \%)$ (Figure 3). Thus, it seems that the use of eGSS for the diagnosis of the injury stage is not the most appropriate of the 3 ways to classify the visual field. 


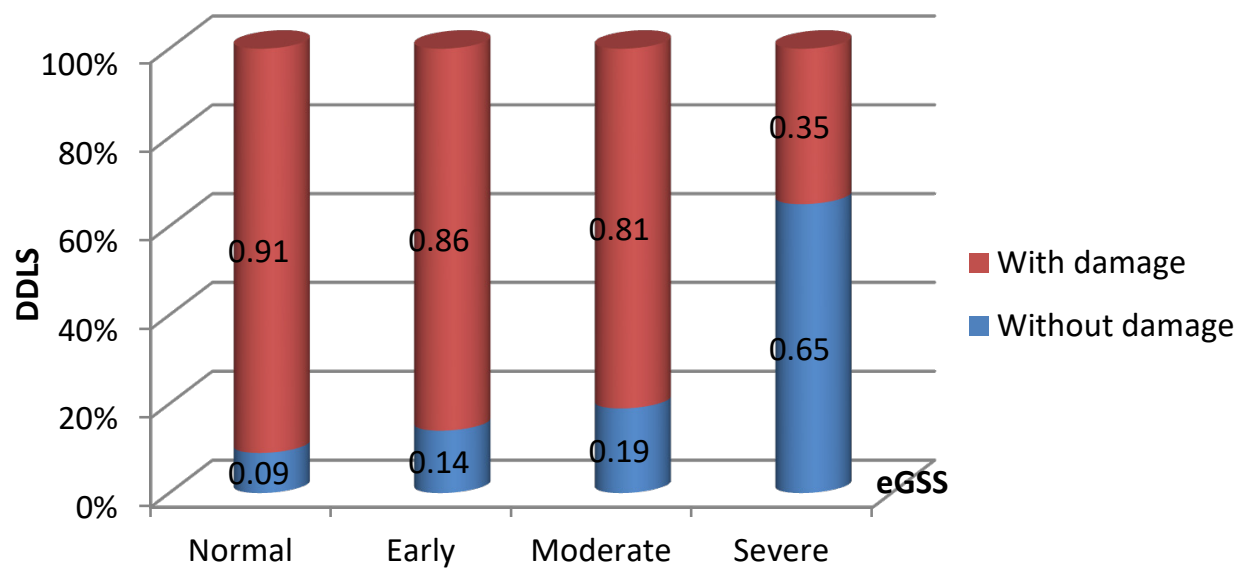

Figure 3 Comparison between eGSS and DDLS.

Classifying mGSS based on VFI index evaluation in the VF, the advantage were found to be that mGSS was could accurately diagnose early and severe stages. It is simple and easy to use in clinical settings. The downside is that it can only be used with improved Humphrey perimeters, which cannot be used in the 10-2 and, for MD $<-20 \mathrm{~dB}$ cases; the value of VFI could be frozen because of any calculation [3]. Visual field was transferred from the deviation scale to local deviation. In Figure 4, mGSS, as well as HPA, had disagreements with DDLS but with a low rate: $87 \%$ of normal visual field cases on mGSS also exhibited normal neuro optic head damage on DDLS, and $89 \%$ of head. Economic damage had serious visual field damage on mGSS. Although it had the same ability to differentiate stages like HAP, mGSS had a simpler classification ability, easier to apply clinically than HPA.

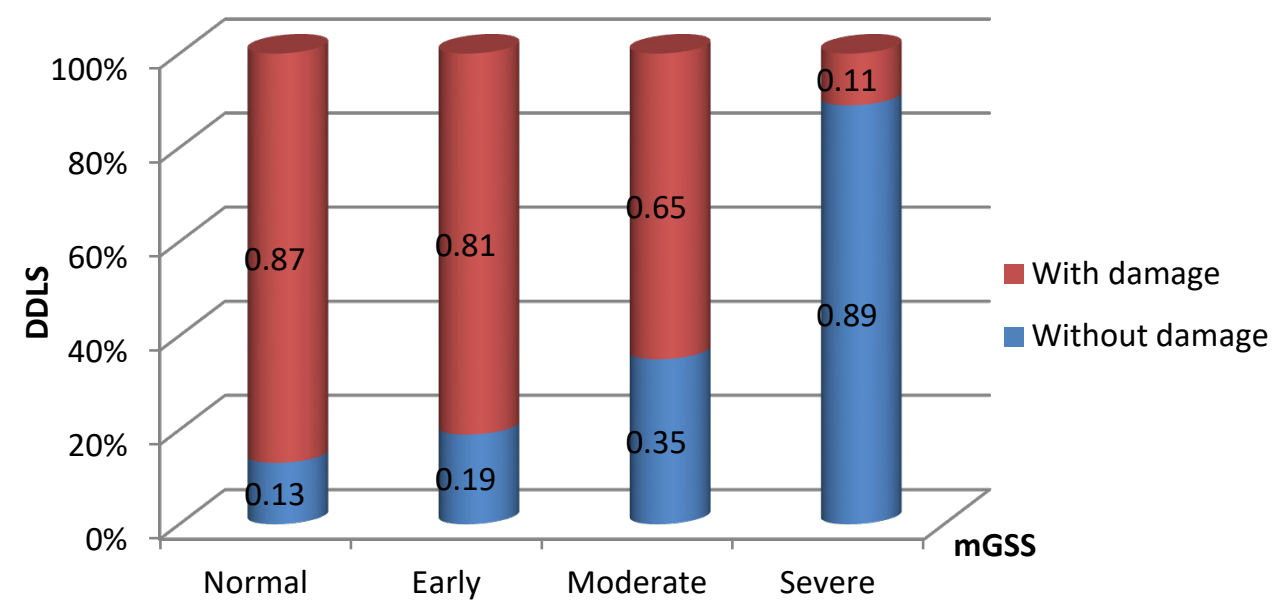

Figure 4 Comparison between mGSS and DDLS. 
http://wjst.wu.ac.th

The results showed that eGSS disagrees with DDLS most in both definite diagnosis and stage diagnosis. In addition, unlike HPA and mGSS, eGSS was completely dependent on MD and PSD, which might have resulted in short-term fluctuations of visual field determination that led to deviations in the results. In addition, eGSS could not determine the shape, location, or depth of injury. Meanwhile, HPA and mGSS were concerned about visual field vulnerability in both peripheral and central areas (nose, the 2 halves of the visual field) and assessed based on each site's vulnerability threshold. Although HPA was consistent with DDLS, it is too simple; only 3 stages are insufficient to assess small changes in visual field vulnerability, HPA is not suitable for use in glaucoma follow-up. While eGSS was simple to use, it does not have a definite diagnostic ability, and tends to estimate disease to be more serious than it actually is. These results suggest that clinical mGSS should be used, due to its ability to diagnose stages and track well. However, there is no perfect classification, so when diagnosing and classifying visual field, including using mGSS, it might still be combined with evaluation of visual field records (gray scale, local deviation) and history [14]. Use of the glaucoma visual field diagnostic criteria of Anderson and Patella is also recommended.

\section{Conclusions}

mGSS and HPA tended to show stronger agreement with standard classifications than eGSS. Due to its simplicity and accuracy, mGSS is recommended for use in clinical practice.

\section{Acknowledgements}

We would like to thank the sponsorship of the Vietnam National Institute of Ophthalmology, grant number 160/QD-BVMTW. We would also like to thank Hanoi Medical University, who collaborated on the topic.

\section{References}

[1] LA Kerrigan-Baumrind, HA Quigley, ME Pease, DF Kerrigan and RS Mitchell. Number of ganglion cells in glaucoma eyes compared with threshold visual field tests in the same persons. Invest. Ophthalmol. Vis. Sci. 2000; 41, 741-8.

[2] PH Artes, N O'Leary, DM Hutchison, L Heckler, GP Sharpe, MT Nicolela and BC Chauhan. Properties of the statpac visual field index. Invest. Ophthalmol. Vis. Sci. 2011; 52, 4030-8.

[3] B Bengtsson and A Heijl. A visual field index for calculation of glaucoma rate of progression. Am. J. Ophthalmol. 2008; 145, 343-53.

[4] P Brusini and S Filacorda. Enhanced Glaucoma Staging System (GSS 2) for classifying functional damage in glaucoma. J. Glaucoma. 2008; 15, 40-6.

[5] HJ Chung, JH Choi, YC Lee and SY Kim. Effect of cataract opacity type and Glaucoma severity on visual field index. Optom. Vis. Sci. 2016; 93, 575-8.

[6] C Cook and P Foster. Epidemiology of glaucoma: What's new? Can. J. Ophthalmol. 2012; 47, 2236.

[7] DW Heo, KN Kim, MW Lee, SB Lee and CS Kim. Properties of pattern standard deviation in openangle glaucoma patients with hemi-optic neuropathy and bi-optic neuropathy. PloS One 2017; 12, e0171960.

[8] I Kocak, M Zulauf and O Bergamin. Evaluation of the Brusini glaucoma staging system for typing and staging of perimetric results. Ophthalmologica 1998; 212, 221-7.

[9] R Malik, WH Swanson and DF Garway-Health. The 'structure-function' relationship in glaucomapast thingking and current concepts. Clin. Exp. Ophthalmol. 2012; 40, 369-80.

[10] M Ng, PA Sample, JP Pascual, LM Zangwill, CA Girkin, JM Liebman, RN Weinreb and L Rocette. Comparison of visual field severity classification systems for glaucoma. J. Glaucoma. 2012; 21, 551-61. 
http://wjst.wu.ac.th

[11] L Racette, FA Medeiros, LM Zangwill, D Ng, RN Weinreb and PA Sample. Diagnostic accuracy of the Matrix 24-2 and original N-30 frequency-doubling technology tests compared with standard automated perimetry. Invest. Ophthalmol. Vis. Sci. 2008; 49, 954-60.

[12] GL Spaeth. The disk damage likelihood scale. Glaucoma Today, Bryn Mawr Communications, 2005.

[13] JR Susanna and RM Vessani. Staging glaucoma patient: Why and how? Open Ophthalmol. J. 2009; 3, 59-64.

[14] RM Vessani and RJ Susanna. University of São Paulo Glaucoma Visual Field Staging System (USP-GVFSS): A new way to stage visual field in glaucoma. Invest. Ophthalmol. Vis. Sci. 2009; 50, 5287. 